\title{
LA TÉCNICA DE VIÑETA Y SU APLICACIÓN EN INVESTIGACIONES EN ENFERMERÍA
}

\section{THE VIGNETTE TECHNIQUE AND ITS APPLICATION TO NURSING RESEARCH}

\author{
Rodrigo Yáñez GaLlardo* \\ Héctor Ahumada Alvarado ** \\ ELIAS Rivas AgUAYO ${ }^{* * *}$
}

\begin{abstract}
RESUMEN
En enfermería es central ejercer una práctica reflexiva, evaluar permanentemente la toma de decisiones clínicas y de gestión, identificando y comprendiendo los razonamientos, las actitudes y valores que las sustentan. Las viñetas son una potente técnica de recolección de datos, que facilita la investigación de dichos procesos y que ha sido escasamente utilizada en nuestro medio. El objetivo de este artículo es presentar una revisión bibliográfica actualizada de esta técnica, identificando su uso en los distintos enfoques metodológicos, describiendo cómo se construyen, presentan y aplican. Se analizan sus principales características, destacando su flexibilidad e idoneidad para el análisis de contenido con énfasis en los factores contextuales. Además, se evalúan su validez y confiabilidad, como también las ventajas y desventajas de su uso en los servicios de salud.
\end{abstract}

Palabras clave: Investigación en enfermería, metodología, viñeta.

\begin{abstract}
In nursing, it is vital to carry out a thoughtful practice and a constant evaluation of clinical and management decisions by identifying and understanding the reasoning, attitudes and values that support them. Vignettes are a powerful data collection technique which facilitates research on such processes and which has been barely used in our field. The purpose of this paper is to present an up-to-date bibliographical overview of this technique. Its use, construction, presentation and application from the diverse approaches will be described. Its main features will be analyzed and two of them will be emphasized: its flexibility and suitability for content analysis focusing on contextual factors. In addition, its validity and reliability as well as the advantages and disadvantage of its use in health services will be evaluated.
\end{abstract}

Key words: Nursing research, methodology, vignettes.

Fecha recepción: 13/12/11 Fecha aceptación: 30/11/12

\footnotetext{
* Psicólogo. Docente Departamento de Psicología, Facultad de Ciencias Sociales, Universidad de Concepción, Concepción. Chile.E-mail: ryanez@udec.cl

${ }^{* *}$ Psicólogo. Consejo de Defensa del Niño (CONEDI). Programa de Salidas Alternativas “Pewungen”, Osorno. Chile. E-mail: hahumada@udec.cl

“** Psicólogo. Centro Comunitario Luterano Agüita de la Perdiz. Coordinador Programas "Niñez, Juventud y Familia” y

"Formación Interna". Chile. E-mail: erivas40@gmail.com
} 


\section{INTRODUCCIÓN}

Es inherente a la práctica de la enfermería que en forma habitual se deban tomar decisiones complejas, relacionadas a su quehacer $\mathrm{y}$, por tanto, dichos profesionales deben mostrar una alta competencia en pensamiento crítico y reflexivo, permitiéndoles un examen riguroso de evidencias y asociarlo a conocimientos, razonamientos y actitudes propios y explorar marcos de referencia alternativos $(1,2)$. El pensamiento reflexivo en enfermería es central si se avizora la emergencia de un mundo postmoderno, donde el cuidado de la salud muestra rápidos y profundos cambios y las(os) enfermeras(os) son crecientemente confrontados no sólo a problemas clínicos complejos sino, especialmente, con dilemas éticos (3). Dado este contexto, es muy necesario contar con una técnica de recolección de datos que permita explorar cómo se toman decisiones frente a temas sensibles e identificar y comprender los procesos cognitivos y afectivos involucrados, explorar el grado de consenso existente entre los profesionales, las variaciones existentes, así como también determinar cómo influyen los factores contextuales en sus decisiones (4).

Las viñetas son una técnica de recolección de datos que consiste en una breve historia, una descripción corta de una situación y de personas hipotéticas que simulan la experiencia de la vida real y que contiene la información necesaria para que los entrevistados puedan emitir un juicio o tomar una decisión sobre la situación descrita $(4,5)$. Por tanto, se puede considerar la respuesta a una viñeta como la elaboración de un significado situado ante, generalmente, una situación compleja. Su uso surge de las limitaciones que presentan las técnicas de recolección de datos tradicionales: a) con las respuestas a las encuestas y la observación directa no es posible conocer el razonamiento de las personas
(6); b) la técnica de la entrevista no permite una estandarización de la presentación de un estímulo complejo y un análisis de los procesos cognitivos y emocionales involucrados en los razonamientos de los participantes.

En el ámbito de la enfermería, recién en la década del 90 se aprecia un mayor uso de las viñetas, no obstante, en Latinoamérica han sido escasamente utilizadas $(6,7)$. Las viñetas se han utilizado en diversas áreas de la enfermería. En educación en salud se ha evaluado la práctica reflexiva de las(os) futuras(os) profesionales; por ejemplo, en una investigación se evaluó en estudiantes de enfermería, sus competencias para evaluar e intervenir pacientes con embolia pulmonar (1). Se ha estudiado el juicio clínico, por ejemplo identificando los factores que enfermeras/os consideran para tomar decisiones sobre cuándo administran un medicamento a un paciente (8). Un área especialmente fructífera de investigación ha sido la toma de decisiones ética de las enfermeras, donde se ha estudiado tanto la habilidad de reflexión crítica como la conducta ética de dichos profesionales (3). Un reciente estudio constata que existe continuo énfasis en los aspectos técnicos de la enfermería que frecuentemente oscurecen la preocupación por los aspectos éticos de la práctica, e indica que los profesionales de enfermería tienen dificultades en la toma de decisiones éticas en situaciones complejas, mostrando tendencia a guiarse por un pensamiento apegado a las convenciones y reglas y escaso uso de la reflexividad crítica (9).

Dada la utilidad de las viñetas para dirigir investigaciones sobre toma de decisiones en enfermería y constatando que existen escasas descripciones del uso de esta técnica de investigación en nuestro medio, el propósito de este trabajo es identificar los principales objetivos de su uso según el paradigma metodológico, describir cómo se construyen y aplican, y analizar sus ventajas y desventajas. 


\section{ENFOQUES METODOLÓGICOS EN EL USO DE LAS VIÑETAS}

La viñeta puede ser utilizada desde el paradigma metodológico cuantitativo o cualitativo. Desde la metodología cuantitativa, posterior a que los participantes lean la viñeta se incluye una encuesta con alternativas de respuestas (escala Likert). Una ventaja de esta alternativa es que resulta posible aplicarlo simultáneamente a una gran cantidad de personas. De esta forma, se pueden realizar estudios descriptivos de, por ejemplo, el grado de consenso existente entre enfermeras sobre diversas decisiones (7).

Más complejo y de índole experimental, consiste en utilizar un diseño factorial con las viñetas, lo cual implica combinar la técnica de encuesta y el diseño experimental. En este caso, el objetivo suele ser determinar en qué grado algunos factores influyen en una decisión que a los encuestados se les plantea al final de una viñeta. En este caso, primero se definen como mínimo 2 variables independientes (factores) y el investigador manipula la construcción de la viñeta incorporando los niveles de las variables independientes. Por ejemplo, en una investigación una variable independiente podría ser sexo, se construye entonces dos viñetas, en una el personaje principal será hombre y en otra viñeta será mujer. Paso siguiente, se podrían formar dos grupos seleccionando sus integrantes al azar y se aplicaría una viñeta a un grupo y la otra viñeta a otro grupo. Utilizando esta metodología es posible establecer el efecto de cada variable independiente en los juicios o decisiones del encuestado (variable dependiente) y, además, se puede examinar el efecto interactivo entre las variables independientes $(4,7)$. De este modo, se puede explicar y predecir los juicios o decisiones tomadas por los encuestados a partir de las variables independientes, lográndose controlar en alto grado otras variables asociadas a los participantes (per ejemplo, rasgos de perso- nalidad), dado que los participantes fueron seleccionados al azar (10).

Si el uso de la viñeta se ajusta a la metodología cualitativa, posterior a la viñeta se planteará una pregunta abierta y se realizará una entrevista en profundidad o semiestructurada, pudiéndose realizar estas actividades en forma individualizada o grupal $(10,11)$. En este caso, se deja que el participante defina la situación en sus propios términos y se le pregunta qué haría en esa situación o cómo cree que lo haría una tercera persona, dependiendo del objetivo de la investigación. El objetivo de su uso sería comprender el sistema de creencias del participante, que está en la base de sus decisiones clínicas y de gestión.

\section{MÉTODOS DE CONSTRUCCIÓN, PRESENTACIÓN Y APLICACIÓN DE LAS VIÑTTAS}

Se pueden distinguir tres métodos en su construcción (12). La primera, se llama foto instantánea y consiste en la recopilación de información a partir de la observación de campo. Este método también es denominado natural, ya que pesquisa las problemáticas y las historias en el campo mismo de manera fenomenológica. La segunda, se llama retrato y se intenta representar a los participantes de la situación, tanto sus características personales, sus conductas y sus experiencias, basándose fundamentalmente en lo que dicen los propios actores. Este procedimiento es menos natural que el anterior, presenta mayores contaminaciones para pesquisar una situación, ya que el investigador necesariamente filtrará las acciones, palabras y comportamiento no-verbal que le interesen. Por último, está el método compuesto, que es una mezcla de diversas situaciones que pueden ser tanto reales como ficticias, observadas o hipotéticas.

Las viñetas se pueden presentar en diferentes formatos, los más frecuentes son una 
historia escrita; sin embargo, también se puede utilizar un video o fotografía (11). Pudiéndose presentar en papel, cinta de video y/o a través de computadoras (13). A esto se suman los avances tecnológicos actuales que permiten pensar en la posibilidad de crear escenarios más complejos utilizando tecnología como la de los videojuegos e imágenes en 3 dimensiones (10).

Con respecto a su aplicación, ésta se puede realizar en forma individual o grupal. Esta última alternativa tiene la ventaja de estimular la discusión.

\section{CARACTERÍSTICAS DE LAS VIÑETAS}

Una de las características más importante de esta técnica es que es posible estudiar el comportamiento humano en su propio contexto. La viñeta intenta ser una representación lo más fidedigna posible de una cierta situación, factible de suceder en la realidad (14). Al representar una situación contextualizada, se pretende que los encuestados logren una comprensión precisa acerca de una situación. Así, permitiría poner en evidencia las acciones y/o los mecanismos cognitivos que son generalmente utilizados al afrontar las situaciones planteadas. Por lo tanto, la situación problematizada en una viñeta permite al investigador conocer cómo las personas experimentan los sucesos y obtener de los participantes sus percepciones, conocimiento y actitudes relacionados a una situación descrita (15).

Un mecanismo cognitivo que subyace al uso de la viñeta es la proyección (16). Si bien esta técnica intenta representar una situación cotidiana lo más cercanamente posible a la realidad, también debe contener un cierto grado de ambigüedad, con el fin de que la persona pueda mostrar aspectos de sí misma que generalmente no evidencie, lo que permite enriquecer la respuesta. Así, esta ambigüedad con que se redacte un relato en una viñeta permitirá acceder a otro tipo de información relevante acerca del mismo sujeto (14). El mecanismo de la proyección se activará, especialmente, si la persona se pone en el lugar del protagonista del relato, vale decir, si se identifica con este y proyecta en él sus características personales como, por ejemplo, sus respuestas emocionales, las cuales no son en todo momento fácil de manifestar (10).

Hay consenso entre los investigadores $(11,13,14)$ en que las viñetas deben poseer las siguientes 3 cualidades:

a) Las historias deben ser fáciles de entender; si bien deben tener suficiente información contextual para que todos los participantes entiendan las situaciones que se les proponen, no deben ser demasiado complejas;

b) Deben atraer el interés y estimular la imaginación. Se debe hacer coincidir las características de las viñetas con las características del grupo de participantes y

c) Debe hacerse acorde con las capacidades cognitivas que posean los participantes.

Además, la construcción de las viñetas es flexible; éstas pueden variar en el número que se les presenta a los participantes, pueden ser cortas o largas, simples o complejas. Esta flexibilidad no significa que no se deben tener variadas consideraciones a la hora de construir las mismas. Los atributos de la viñeta, por ejemplo su longitud y complejidad, la dificultad de la tarea, número y dificultad de las palabras, influirán significativamente en la calidad de las respuestas (11).

Un factor crucial en la construcción de una viñeta es la verosimilitud, es decir mientras más plausibles y cercanos a la realidad sean vistos los escenarios presentados a los participantes más propensos son de generar información valiosa $(10,11)$. Una vez construida la viñeta, para asegurar la verosimilitud una alternativa es realizar pruebas piloto, con una posterior adaptación que intente 
reelaborar los escenarios vistos como poco plausibles. La realización de pruebas piloto permitirá principalmente lograr tres objetivos: asegurar que las viñetas tienen un significado equivalente en relación a la cultura de los participantes; que todas las personas entienden la viñeta de la misma manera y garantizar un número suficiente de viñetas que permitan abordar de manera suficiente el fenómeno (17).

Aunque se recomienda el uso de varias viñetas, una dificultad al utilizarlas es que éstas deben ser equivalentes, es decir que los dominios representados en cada una se entienden de la misma manera por todos los encuestados, independiente de su edad, sexo, ingresos, educación, país de residencia u otras variables sociodemográficas (17). Para lograr esto puede ser útil incluir términos concretos en lugar de frases vagas que están sujetos a diferentes interpretaciones.

\section{VALIDEZ Y CONFIABILIDAD DE LAS VIÑETAS}

Pese a la amplia revisión bibliográfica realizada, se ha encontrado que son escasos los estudios sobre validez y confiabilidad de esta técnica, la mayoría incluye recomendaciones más que procedimientos rigurosos referidos a esta temática. Un criterio de validez es construir una situación ficticia que represente una escena cotidiana de la vida de las personas, para que éstas puedan ver reflejada lo más cercanamente posible su propia experiencia (12). Para asegurarse de que esto se cumpla en la viñeta, se puede, una vez construida la viñeta, mostrarla a las personas que participarán del procedimiento o a las que no participarán, pero que se relacionan directamente con la situación y que comparten algunos atributos similares como: profesión, sexo, edad, entre otros (17).

La aplicación test-retest ofrece una medida clave de la confiabilidad de la viñeta, en la medida que las respuestas individuales puedan ser estables en el tiempo (17). Estas pruebas han mostrado que el nivel de acuerdo es razonablemente alto entre diferentes poblaciones y existe escasa variación a través de distintas viñetas, en particular en un mismo dominio de interés.

\section{PRINCIPALES VENTAJAS Y DESVENTAJAS EN EL USO DE VIÑETAS EN LOS SERVICIOS DE SALUD}

a) Ventajas. Dado que las viñetas tienen un carácter "hipotético" que involucra a un "otro ficticio", posibilita obtener información que va más allá de la situación personal actual del informante, favoreciendo la despersonalización en las respuestas de los participantes (13). Lo anterior es particularmente útil porque facilita que los participantes se expresen libremente sobre temas complejos, socialmente sensibles, ayudando a que se distancien de un tema potencialmente difícil para referirse ante extraños y puedan expresarse abiertamente $(13,15)$. Vinculado a lo anterior, las viñetas ayudarían a reducir las respuestas socialmente deseables, esto se logra cuando se le pide a los participantes que asuman el rol de un personaje en vez de responder desde un punto de vista personal, creando un efecto de distancia entre la vida real del participante y el contexto presentado en las viñetas (13). Con esto los funcionarios pueden expresar sus propias percepciones sobre temas muy familiares, pero permanecer ajenos a ellos, no poniendo en riesgo la devaluación de su imagen personal, dando respuestas más sinceras.

Otra ventaja de la técnica, no es de extensa aplicación, ni requiere gran habilidad de quienes la administran y los participantes no necesitan de un conocimiento profundo sobre el tema a tratar, sólo demandaría claridad conceptual, habilidades de edición de los investigadores y una relación de colaboración 
entre los investigadores y los funcionarios (15).

Finalmente, las viñetas gracias a la naturaleza del "relato" suelen ser relajantes, agradables, interesantes y puede reducir la sensación de sobrecarga que genera el proceso de entrevista. Al mismo tiempo pueden promover la reflexión y el pensamiento crítico $(5,17)$.

b) Desventajas. La principal crítica es determinar si los participantes reaccionan o se comportan de manera similar en la vida real como declaran al analizar las historias. En otras palabras, es el problema de validez predictiva (11). Esta limitación se encuentra principalmente en el momento de la interpretación y generalización de los resultados. Dado que las viñetas no pueden tener en cuenta todos los posibles factores del medio ambiente o personales que pueden realmente influir en las decisiones de una persona en la vida real, esta técnica no proporciona información directa sobre las respuestas de los encuestados en situaciones reales (11). Es decir, los investigadores deben ser cuidadosos al momento de realizar generalizaciones a partir de los datos (15). Debido a esta complejidad para generalizar fuera del contexto particular de la viñeta algunos estudios reportan datos y análisis dentro de los límites de cada escenario. Sin embargo, cuando las respuestas son congruentes con la información obtenida de otras fuentes, es posible realizar generalizaciones (13).

En relación al análisis de las respuestas, desde la metodología cualitativa, está el problema que existen múltiples formas de interpretar tanto las viñetas, por parte de los informantes, como las respuestas, por parte de los investigadores. A veces, el no querer inducir a los participantes puede llevar a la obtención de respuestas inconsistentes con el tema en estudio, las cuales, si bien pueden ofrecer información valiosa, quedarán en un plano secundario dentro de la investigación. Debido a esto, resulta un gran desafío inter- pretar las palabras de los informantes de manera de representar fielmente su perspectiva, sin embargo, el aceptar las limitaciones de este proceso no implica que no se logre una interpretación útil de la experiencia (5).

\section{REFERENCIAS}

1. Fero LJ, O’Donnell JM, Zullo TG, Dabbs $\mathrm{AD}$, Kitutu J, Samosky JT et al. Critical thinking skills in nursing students: comparison of simulation-based performance with metrics. J Adv Nurs. 2010; 66(10): 2182-93.

2. Eterovic C, Stiepovich J. Enfermería basada en la evidencia y formación profesional. Cien enferm. 2010; XVI (3): 9-14.

3. Dierckx de Casterlé B, Izumi S, Godfrey NS, Denhaerynck K. Nurses' responses to ethical dilemmas in nursing practice: meta-analysis. J Adv Nurs. 2008; 63(6): 540-9.

4. Brauer P, Hanning RM, Arocha JF, Royall D, Goy R, Grant A, et al. Creating case scenarios or vignettes using factorial study design methods. J Adv Nurs. 2009; 65(9): 1937-45.

5. Schoenberg, N. \& Ravdak H. Using vignettes in awareness and attitudinal research. Int J Soc Res Methodol. 2000; 3(1): 63-74.

6. Galante AC, Aranha JA, Beraldo L, Pelá NT. A vinheta como estratégia de coleta de dados de pesquisa em enfermagem. Rev Lat Am Enfermagem. 2003; 11(3): 357-63.

7. Ludwick R, Wright ME, Zeller RA, Dowding DW, Lauder W, Winchell J. An improved methodology for advancing nursing research: factorial surveys. ANS Adv Nurs Sci. 2004; 27(3): 224-38.

8. Van Hulle Vincent C, Gaddy EJ. Pediatric nurses' thinking in response to vignettes on administering analgesics. Res Nurs Health. 2009; 32(5): 530-9. 
9. Goethals S, Gastmans C, Dierckx de Casterlé B. Nurses' ethical reasoning and behaviour: A literature review. Int J Nurs Stud. 2010; 47: 635-50.

10. Jenkins N, Bloor M, Fischer J, Berney L, Neale J. Putting it in context: The use of vignettes in qualitative interviewing. Qual Res. 2010; 10(2): 175-98.

11. Petelewicz M. The successful story? applicability of the vignette technique. II Conferencia sobre Métodos Cualitativos de Investigación; mayo 2008; Vaasa, Finlandia.

12. Spalding NJ, Phillips T. Exploring the use of vignettes: From validity to trustworthiness. Qual Health Res. 2007; 17(7): 954-62.

13. Hughes R, Huby M. The application of vignettes in social and nursing research. J Adv Nurs. 2002; 37(4): 382-6.
14. Barter C, Renold E. The use of vignettes in qualitative research. Social Research Update. 1999; 25: 1-4.

15. Paddam A, Barnes D, Langdon D. Constructing vignettes to investigate anger in multiple sclerosis. Nurse Res. 2010; 17 (2): 60-73.

16. Richman J, Mercer D. The vignette revisited: Evil and the forensic nurse. Nurse Res. 2002; 9(4): 70-82.

17. Salomon JA, Tandon A, Murray CJL. Using vignettes to improve cross-population comparability of health surveys: Concepts, design and evaluation technique. Global Programme on Evidence for Health Policy Discussion Paper, Geneva: World Health Organization [Internet]. 2001 [citado 9 de noviembre de 2011]. Disponible en: http://cdrwww.who.int/ healthinfo/paper41.pdf 